(2) Open Access Full Text Article

REVIEW

\title{
Diagnosis and treatment of multiple sclerosis in pediatric and adolescent patients: current status and future therapies
}

This article was published in the following Dove Press journal:

Adolescent Health, Medicine and Therapeutics

29 July 2010

Number of times this article has been viewed

\section{E Ann Yeh}

Department of Neurology, Pediatric MS Center of the JNI, SUNY Buffalo, Buffalo, NY, USA
Correspondence: E Ann Yeh WCHOB, Division of Child Neurology, Pediatric MS Center of Excellence, 219 Bryant St, Buffalo, NY 14222, USA $\mathrm{Tel}+\mathrm{I}-7 \mathrm{l}$ 6-878-7840

Fax +I-7| 6-878-7236

Email ayeh@thejni.org
Abstract: Pediatric-onset multiple sclerosis (MS) comprises approximately $3 \%-5 \%$ of cases of MS in North America. Recent years have seen significant advances in the diagnosis and treatment of this condition, including the introduction of proposed diagnostic criteria for pediatric demyelinating disorders, and a growing body of knowledge regarding treatment options. This article reviews current approaches to the diagnosis and management of pediatric MS.

Keywords: pediatric, therapy, interferon, glatiramer acetate, chemotherapy, breakthrough disease, definitions, MRI

Pediatric-onset multiple sclerosis (MS) comprises approximately 3\%-5\% of cases of MS in North America. ${ }^{1-4}$ Recent years have seen significant advances in the diagnosis and treatment of this condition, including the introduction of proposed diagnostic criteria for pediatric demyelinating disorders, and a growing body of knowledge regarding treatment options. This article will review current approaches to the diagnosis and management of pediatric MS.

\section{Diagnosis: clinical and magnetic resonance imaging criteria}

Growing evidence in adult MS research suggests benefit to early treatment using disease-modifying therapies (DMTs). As such, making a timely diagnosis of MS is essential. In pediatric MS, this is particularly in immediate need because recent research points to more aggressive disease in this group, with more frequent relapses ${ }^{5}$ and higher T2 lesion load on brain magnetic resonance imaging (MRI), both early and later in the disease. ${ }^{6,7}$ Importantly, however, not all first-time demyelinating episodes in children will become multiple sclerotic. Indeed, although $45 \%$ of all children with a first-time demyelinating episode will later receive a diagnosis of $\mathrm{MS},{ }^{8}$ only one-fifth of children with a first-time episode of acute disseminated encephalomyelitis (ADEM) will eventually be diagnosed with MS. ${ }^{9}$

Recently, formulated diagnostic criteria for pediatric MS may help to improve diagnostic accuracy for the clinician. According to definitions of the International Pediatric MS Study Group (IPMSSG) published in $2007,{ }^{10}$ pediatric MS may be diagnosed after two clinical episodes of central nervous system (CNS) demyelination that are separated by at least 30 days. No lower age limit is specified (Table 1).

According to these definitions, the Barkhof adult brain MRI criteria can be used to meet the requirement for dissemination in space by demonstrating three of the 
Table I Diagnostic criteria for pediatric MS

\begin{tabular}{l}
\hline Pediatric MS diagnostic criteria \\
\hline - Dissemination in space and time \\
- 2 episodes of demyelination \\
- Separated by $30 \mathrm{~d}$ \\
- No lower age limit \\
- Dissemination in space \\
- MRI: Barkhof criteria (see Table 2) \\
- CSF + 2 MRI lesions \\
- $\geq 2$ oligoclonal bands OR \\
- Elevated IgG index \\
- Dissemination in time \\
- MRI: new T2 lesions or gadolinium enhancing \\
lesions $>3$ mo after initial presentation \\
\hline
\end{tabular}

Abbreviations: MS, multiple sclerosis; MRI, magnetic resonance imaging; CSF, cerebrospinal fluid; IgG, immunoglobulin G.

following four features: (1) nine or more white matter lesions or one gadolinium enhancing lesion, (2) three or more periventricular lesions, (3) one juxtacortical lesion, and (4) an infratentorial lesion. The combination of an abnormal CSF and two lesions on MRI, of which one must be in the brain, can also meet the dissemination in space criteria. The CSF must show either at least two oligoclonal bands (OCB) or an elevated IgG index. The MRI may also be used to satisfy criteria for dissemination in time following the initial clinical event, even in the absence of a new clinical demyelinating event; new T2-bright or gadolinium-enhancing foci must develop 3 or more months following the initial clinical event.

These definitions are currently under review. The adult MRI criteria mentioned above have been found to have low sensitivity and specificity in pediatric MS. ${ }^{11}$ In response to this, several groups have proposed MRI diagnostic criteria for pediatric MS. According to one set of criteria, more than two of the following criteria must be satisfied: $\geq 5 \mathrm{~T} 2$ lesions, two periventricular lesions, or one brainstem lesion. The same group has proposed the following criteria for differentiating
ADEM from MS, including two or more of the following: absence of diffuse bilateral lesion pattern, black holes, or greater than two periventricular lesions. ${ }^{12,13}$ The ADEM criteria have been found to be highly sensitive (99\%) and relatively specific (75\%) in differentiating MS from ADEM when evaluated using an outside cohort of children with known MS (Table 2). ${ }^{14}$

\section{Pathophysiology}

Pathologic investigations have suggested that MS lesions in the adult population are heterogeneous; although some lesions may show T-cell mediated and antibody-mediated loss, other lesions may show oligodendrocyte dystrophy suggestive of viral-mediated or toxinmediated demyelination. ${ }^{15}$ Evaluation of childhood MS is complicated by presentation with confluent lesions on MRI, particularly in younger children who may initially be diagnosed with ADEM. ${ }^{16}$ Thus, distinguishing a first attack of MS from the monophasic condition, ADEM is often challenging. Recent work on children and adults with MS and ADEM suggests pathologic overlap between the two: perivenous inflammation was found to be a hallmark of ADEM but was also seen in a small subset of patients with MS. ${ }^{17}$

MS is believed to be the result of early triggering events to CNS self-antigens in genetically predisposed individuals. ${ }^{18}$ Antimyelin antibodies have been investigated in the adult MS population as a marker for MS. ${ }^{19}$ Recent work suggests that myelin basic protein antibodies in the serum and CSF of children may modulate the clinical presentation of MS in children and are associated with an ADEM-like presentation, suggesting a role for humoral immunity in this group..$^{20}$ Further evidence for the involvement of innate immunity in this population can be seen in recent work showing increased number of white blood cells and neutrophils in the CSF of younger children with MS compared with older children. ${ }^{21}$

Table 2 Barkhof (adult MS) and 2 other recently proposed sets of diagnostic criteria for pediatric MS

\begin{tabular}{lll}
\hline MRI criteria for pediatric MS & \\
\hline Barkhof criteria & MS vs other & MS vs ADEM \\
$\bullet \geq 9$ white matter lesions or one gadolinium & nondemyelinating diseases: $>2$ of & $\bullet$ Absence of diffuse bilateral lesion \\
enhancing lesion & $\bullet \geq 5$ T2 lesions & $\bullet 2$ periventricular lesions \\
$\bullet \geq 3$ periventricular lesions & $\bullet$ I brainstem lesion \\
- One juxtacortical lesion & \\
\hline One infratentorial lesion & \\
\hline
\end{tabular}

Note: The new criteria have been found to be sensitive and specific in the diagnosis of pediatric MS. ${ }^{12,13}$

Abbreviations: MS, multiple sclerosis; MRI, magnetic resonance imaging; ADEM, acute disseminated encephalomyelitis. 
Taken together, these studies suggest the possible importance of B cell-mediated processes in childhood MS, particularly in the younger subset of patients with an ADEMlike presentation.

\section{Therapies}

\section{Treatment of exacerbations}

\section{Steroids}

Randomized, controlled trials of therapy for acute MS exacerbations in children have not been conducted. Clinical practice in the pediatric population is therefore based largely on adult practices. Importantly, not all children experiencing episodes of acute demyelination receive treatment for the exacerbations. If symptoms are mild and do not cause impairment, the decision to provide only supportive care may be made by some practitioners. However, it is clear that there is a need for knowledge regarding treatment for exacerbations in this population, as the annualized relapse rate in children with MS has been found to be almost three-fold higher (1.13 vs 0.40 ) than in adults. ${ }^{5}$

Evidence in adult MS suggests the use of pulse steroids can lead to improved recovery from disability after an acute attack and possibly, a decreased risk for the development of MS in the first 2 years after an episode of optic neuritis. ${ }^{22,23}$ The relative superiority of intravenous (IV) steroids over oral steroids for acute MS attacks has undergone much debate. ${ }^{24}$ Some analyses suggest the two may be equivalent, although heterogeneity between outcome measures and patient populations limit these conclusions. ${ }^{25}$ In adults with MS, a standard dosing recommendation of methylprednisolone $1 \mathrm{~g}$ IV daily for 3-5 days is usually employed. In the pediatric population, a survey of US practitioners suggests that many adhere to the treatment regimen of IV methylprednisolone $20-30 \mathrm{mg} / \mathrm{kg} / \mathrm{d}$ (up to $1 \mathrm{gm}$ ) for 3-5 days for acute MS exacerbations. ${ }^{26}$ The literature in adult MS does not support the need of a steroid taper after completion of pulse steroid therapy, but no evidence is available for the pediatric population.

\section{Intravenous immunoglobulin}

Monthly intravenous immunoglobulin (IVIG) therapy to prevent MS relapses in adults has been evaluated in several trials. ${ }^{27-31}$ Favorable results in preventing progression in primary progressive MS and secondary progressive MS (SPMS) have been found, ${ }^{32}$ but its use in acute MS relapses has only been evaluated in two trials (as an adjunct to intravenous methylprednisolone), where superiority was not demonstrated. ${ }^{33,34}$ However, it may be of benefit in the treatment of corticosteroid refractory acute optic neuritis in adults. ${ }^{35}$ In the pediatric MS population, individual case reports of treatment with IVIG in refractory cases of acute demyelination in children have been published, suggesting possible improvement, although these cases are limited to children with optic neuritis and ADEM. ${ }^{36-38}$

\section{Plasmapheresis}

Plasma exchange is another therapy that may be considered in MS relapses that are corticosteroid refractory. Successful use of plasma exchange for severe episodes of acute demyelination has been described in the adult population..$^{39,40}$ One case report and one series have described a decrease in relapses and clinical improvement in three of four children with pediatric MS, who had severe relapses. ${ }^{41,42}$

\section{Prevention of relapses: DMTs}

Pediatric-onset MS has been associated with significant cognitive impairment ${ }^{43}$ and lesion burden on MRI. ${ }^{7}$ Thus, medical intervention focused on preventing relapses may reduce the neuropsychological burden of MS on this population. Early intervention with DMT in pediatric MS has been demonstrated to result in a decreased likelihood of presentation with a third clinical relapse. ${ }^{44}$

Six DMTs have been approved for the treatment of relapsing-remitting MS (RRMS) in the adult population, including four first-line (glatiramer acetate [GA], intramuscular [IM] and subcutaneous [SC] interferon $\beta$-1a, and SC interferon $\beta-1 b$ ), and two second-line therapies (mitoxantrone and natalizumab). In addition, therapies such as rituximab, daclizumab, and cyclophosphamide have been evaluated in phase II trials in adults with breakthrough disease, as have add-on therapies such as monthly steroids and IVIG. ${ }^{45-50}$ Oral therapies, including cladribine and fingolimod, have also been evaluated in the adult population. ${ }^{51-53}$ This section will review the currently available evidence for the use of these agents in children.

\section{Interferon $\beta$}

Interferon $\beta$ is thought to act in MS via inhibition of proinflammatory cytokines, induction of anti-inflammatory mediators, reduction of cellular migration, and inhibition of autoreactive T cells.$^{54,55}$ Large phase III studies showed that chronic administration of recombinant interferon $\beta$ reduced the number of relapses and slowed the progression of physical disability in adult patients with RRMS. These placebo-controlled studies showed an approximately $30 \%$ reduction in exacerbation (relapse) rate in patients treated for 2-4 years compared with interferon $\beta$ compared with placebo. ${ }^{56}$ 
Several retrospective case series have described the use of interferon $\beta-1 \mathrm{a}$ in the pediatric population. Follow-up in these series has ranged from 12 to 48 months. Although the majority of reports described are of children older than 10 years, Tenembaum et a $\mathrm{l}^{57}$ included eight children younger than 10 years at first injection in their series. Apart from four patients with SPMS reported in Tenembaum et al's article, all patients had RRMS.

Interferon $\beta-1 \mathrm{a}$ and interferon $\beta-1 \mathrm{~b}$ appear to be safe and well tolerated in this population, although discontinuation rates are in the range $30 \%-50 \%{ }^{58-63}$ Many children on interferon $(35 \%-65 \%)$ report flu-like symptoms. Other relatively frequently observed side effects include leukopenia (8\%-27\%), thrombopenia (16\%), anemia (12\%), and transient elevation in transaminases $(10 \%-62 \%) .^{57-59,61}$

Abnormalities in liver function tests (LFTs) may be more pronounced in younger children taking interferon. In one study, $25 \%$ of children (average age of initiation of medication, 14.6 years; range, 8.1-17.9 years) taking interferon $\beta$-1a SC were found to have elevated LFTs. None of these children required discontinuation of therapy. Over two-thirds of these elevations occurred in the first 6 months of therapy. ${ }^{61}$ However, in another study evaluating interferon $\beta-1 \mathrm{~b}$ SC, 8 of 43 patients experienced elevation of LFTs $(>2$ times the upper limit or normal). Importantly, the children with elevated LFTs were predominantly younger than 10 years. Five of eight $(62.5 \%)$ children aged younger than 10 years in this study experienced LFT elevations. Two of these children were on full adult doses ( $8 \mathrm{MIU}$ ), two were on $50 \%$ of the adult dose (4 MIU), and one on one-fourth of the adult dose (2 MIU). By contrast, only $10 \%$ (3/30) of children in this study (older than 10 years of age) suffered from elevated LFTs in the first 6 months of treatment with interferon $\beta-1 b$ SC. ${ }^{59,61}$ Temporary interruption of interferon treatment appears to lead to normalization of LFTs in children and is accompanied by safe reintroduction of therapy after a temporary withdrawal of medication. ${ }^{59,61}$

Given these results, we recommend close LFT monitoring on all children on interferons, particularly in the first 6 months of treatment. Following the practices outlined above, should the LFTs increase to greater than two-fold higher than the upper limit of normal, we suggest that the medication be withheld, the LFTs rechecked within a month, and the medication be reintroduced, initially at a lower dose, after normalization of the LFTs.

Over two-third of children taking the SC formulation of interferon $\beta$-1a have reported injection site reactions. The injection site reactions occur throughout the treatment course in equal proportions. Pohl et al ${ }^{61}$ reported that after a mean follow-up of 1.8 years, children were equally likely to report injection site reactions early on (0-6 months) and later. ${ }^{61}$ Six percent of children on interferon $\beta$ - 1 a SC experienced abscess and $6 \%$ injection site necrosis over an average follow-up of 1.8 years. ${ }^{61}$ Of those on interferon $\beta$ - 1 b, only $20 \%$ older than 10 years of age and $25 \%$ younger than 10 years of age experienced mild injection site reactions (average follow-up of 33.8 months), which did not lead to discontinuation of therapy. ${ }^{59}$

Dosing of interferon $\beta$ is not established in this population. However, most patients tolerate doses titrated following adult protocols, or gradual titration to $30 \mu \mathrm{g}$ once weekly for interferon $\beta$-1a IM and $22 \mu \mathrm{g}$ or $44 \mu \mathrm{g}$ TIW for interferon $\beta$-1a SC. Children older than the age of 10 tolerate full doses of interferon $\beta-1 b$, though decreased tolerance may exist in the younger population. In one study, two of eight children who initiated interferon $\beta-1 \mathrm{~b}$ at $25 \%-50 \%$ of adult doses did not tolerate escalation to full adult doses. Both were aged younger than 10 years. ${ }^{59}$

With respect to efficacy, there have been no randomized controlled trails (RCTs) evaluating efficacy of interferon $\beta$ in the pediatric population. However, in a prospective, open-label study, Ghezzi et $\mathrm{l}^{64}$ followed 52 patients with pediatric-onset MS, who were treated with interferon $\beta$-1a IM, and found a reduction in annualized relapse rate from 1.9 pretreatment to 0.4 after an average of 42 months on therapy. Similarly, Mikaeloff et $\mathrm{al}^{44}$ reporting on 197 children with RRMS on interferon followed for a mean of 5.5 years, found a reduction in risk of MS attack in both the first year of treatment with interferon (hazard ratio $=0.31$; 95\% confidence interval [CI]: $0.13-0.72$ ), as well as over the first 2 years of treatment (hazard ratio $=0.40 ; 95 \%$ CI: $0.20-0.83$ ). After 4 years of follow-up, the annualized relapse rate remained lower, but the $95 \%$ CI was broader due to the smaller sample size, as not all patients had such a long follow-up (hazard ratio $=0.57 ; 95 \%$ CI: $0.30-1.10) .{ }^{44}$ Because $25 \%-30 \%$ of patients in the adult population will have a benign outcome, ${ }^{65,66}$ defined by most as Expanded Disability Status Scale (EDSS) $<3.0$ for greater than 10 years' duration, it is important to keep these natural history studies in mind when considering the effects of any treatment in children without placebo-controlled groups. ${ }^{67}$ No data are available on whether interferon $\beta$ slows down the progression of disability in children. Furthermore, no data on the effect of these medications on MRI are available for the pediatric population. 


\section{Neutralizing antibodies}

It has been noted widely in the adult literature that neutralizing antibodies to interferon may appear after a patient has been treated with interferon. In the adult population, these antibodies are more likely to be seen in interferon preparations that are given SC multiple times a week. ${ }^{68}$ The relationship between titers of neutralizing antibodies and efficacy has not been established in the adult population, although studies have shown a relationship between sustained high titers of these antibodies and ancillary measures of disease activity in adult MS. ${ }^{69}$ Knowledge regarding the impact of interferon-neutralizing antibodies on the efficacy of medication in the pediatric population, however, is even more limited. No studies have comprehensively evaluated the frequency and significance of neutralizing antibodies in the pediatric population.

\section{Glatiramer acetate}

GA is the acetate salt of a mixture of synthetic polypeptides composed of L-alanine, L-glutamic acid, L-lysine, and L-tyrosine. The drug is designed to mimic human myelin basic protein and is postulated to induce myelinspecific response of suppressor $\mathrm{T}$ lymphocytes and to inhibit specific effector $\mathrm{T}$ lymphocytes. ${ }^{70}$ The treatment consists of daily SC injection of $20 \mathrm{mg}$ GA. In a pivotal phase III trial of adult RRMS patients, GA showed a $29 \%$ reduction in the number of relapses in the treated group vs placebo. ${ }^{71}$ Reduction in MRI activity has been shown in a randomized, controlled trial of adults treated with GA vs placebo. ${ }^{72}$ Recent studies in adults have suggested that GA and interferon $\beta$ have similar efficacy on clinical and MRI activity. ${ }^{73}$

Only three retrospective studies have been published evaluating the use of GA in pediatrics. ${ }^{74-76}$ Kornek et al ${ }^{76}$ followed seven patients with pediatric-onset RRMS for 24 months and reported that the medication was well tolerated. Children were aged 9-16 years at the time of GA initiation. Only two of seven patients were relapsefree over the 24-month treatment period, and EDSS was stable in only three of seven children. In two separate papers, Ghezzi et al described 9 and 11 patients on GA. GA was found to be relatively well tolerated, with 3 of 11 patients experiencing side effects (injection site reactions in one and chest pain in another patient).$^{74}$ The mean annualized relapse rate decreased from 2.8 to $0.25 .{ }^{75}$ Conclusions from these studies regarding the efficacy of this medication cannot be drawn, however, given the small numbers.

\section{Treatment failure: second-line therapies}

Treatment failure is a concern in the pediatric MS population. Of 258 children with MS, who were followed by a network of six US Pediatric MS Centers of Excellence, 123 (48\%) were switched from their first therapy. Noncompliance and intolerable side effects represented a significant proportion of these cases: $16 \%$ (42) changed therapies due to noncompliance or side effects, whereas $28 \%$ (72) changed due to breakthrough disease; $4 \%$ (9) discontinued therapy after the first agent. ${ }^{77}$

The definition of treatment failure is challenging; it is the one that has undergone significant debate among practitioners treating adult-onset MS. Given available data regarding the frequency of relapse in MS in the adult population, it is generally accepted that at least 6 months of observation on a given treatment is necessary prior to deeming that treatment to be suboptimal. Intervening factors include the possibility of drug-drug interaction and unacceptable side effects. At present, our practice is to follow these guidelines and observe all patients for 6 months after initiation of therapy before deciding to change therapies.

Consensus criteria for breakthrough disease in pediatric MS do not exist. Some have proposed criteria including increase in relapse number, new or recurrent MRI lesions, and worsening of cognitive or motor disability. ${ }^{78}$ Limitations of this particular approach include the lack of adequate observational time to gauge whether an individual's relapse rate has decreased. Some advocate clinical evaluation every 3-6 months and an annual MRI in the adult population in order to monitor the response to therapy. Given the more frequent relapses seen in the pediatric population, ${ }^{5}$ our practice is to perform MRI scans of the brain on a semiannual basis with clinical visits every 3 months for the first year after diagnosis. Population studies in adults have shown that disease course during the first 5 years of disease is an excellent predictor of future deficits. Therefore, more frequent follow-up in the early course of the disease is warranted.

There is no accepted algorithm for the management of partial responsiveness. However the following steps may be followed:

1. Increase the frequency of interferon $\beta$ therapy (ie, switching from once a week to three times a week or every other day injections). Although some data support a short-term advantage of more frequent dosing on relapses, the magnitude of the advantage is small. ${ }^{99,80}$ This small potential 
advantage must be weighed against the disadvantages associated with frequent SC doses vs IM once a week dose regimens, as more frequent dosing has been associated with high NABs and decreased efficacy according to MRI and clinical parameters. ${ }^{81,82}$

2. Switch from one monotherapy to another. For example, adult patients with sustained positive NAB titers $\geq 20$ may be switched from interferon $\beta$ to GA. Switching from GA to interferon $\beta$ is also an option, particularly given the rapid and robust effect of interferon $\beta$ on inflammatory activity as measured by MRI (21).

3. Add agents to the "platform" interferon $\beta$ or GA therapy, including other immunomodulatory or cytotoxic agents. These agents have not been studied in MS using RCTs. Therefore, their benefit is unclear.

The immunomodulatory and cytotoxic agents, such as natalizamab, cyclophosphamide, mitoxantrone, and rituximab, that have been used in treatment-resistant cases (ie, in pediatric MS) are discussed in the following section.

\section{Natalizumab}

Natalizumab a recombinant, humanized monoclonal antibody binds the $\alpha_{4}$ subunit of $\alpha_{4} \beta_{1}$ (very late antigen-4 [VLA-4]) and $\alpha_{4} \beta_{7}$ integrins (adhesion molecules), hindering the interaction between VLA-4 and its counter-receptor, vascular endothelial adhesion molecule-1. Disruption of these molecular interactions antagonizes the leukocyte-endothelium adhesion processes necessary for efficient migration of leukocytes across the blood-brain barrier endothelium, reducing the recruitment of immune cells into sites of inflammation within the CNS. ${ }^{83}$

The efficacy of natalizumab for treating relapsing MS in adults has been tested initially in a phase II and a small combination (interferon $\beta$ and natalizumab) study. ${ }^{84,85}$ Two pivotal, randomized, placebo-controlled, phase III clinical trials suggest that natalizumab is an effective therapy for RRMS. ${ }^{86,87}$ In the AFFIRM trial, ${ }^{87}$ natalizumab treatment (300 mg, IV infusion, once every 4 weeks) was compared with placebo. In the SENTINEL trial, the combination of natalizumab and interferon $\beta$-1a (natalizumab: $300 \mathrm{mg}$, IV infusion, once every 4 weeks; interferon $\beta$-1a: $30 \mu \mathrm{g}$, IM injection, once weekly) was compared with placebo plus interferon $\beta$-1a. ${ }^{86}$ Both trials demonstrated the efficacy of natalizumab treatment in reducing relapse rate, disease progression, and occurrence of new MRI lesions in MS.
Natalizumab was well tolerated. The adverse events that were significantly more common in the natalizumab group compared with placebo were fatigue and allergic reactions. Importantly, cases of progressive, multifocal leukoencephalopathy, a rare, usually fatal CNS infection caused by the JC polyomavirus, occurred in association with natalizumab therapy in combination with interferon, prompting its withdrawal. ${ }^{88-90}$ After extensive review, the US Food and Drug Administration approved the reintroduction of natalizumab for treating MS. Natalizumab belongs to a promising novel class of therapeutic agents for the treatment of relapsing MS. However because it is associated with a risk for PML, an individualized risk-benefit assessment is necessary prior to initiation of therapy. A high level of clinical vigilance should be maintained during the therapy. Its use currently requires patient and prescriber registration and compliance, following specific guidelines using a risk map (TOUCH program).

There have been two published papers describing the use of natalizamab in the pediatric population; one is a case report of a 12-year-old child with treatment-resistant disease, who responded well to this therapy. ${ }^{91}$ A German group reported on three patients who were selected for this therapy because they suffered either from poorly controlled disease or from adverse effects from first-line therapies. The medication was tolerated well by these children. Follow-up MRI scans, performed every 6 months, showed no enhancing lesions in these patients. ${ }^{92}$

Of 258 patients with pediatric MS followed at the US Pediatric MS Centers of Excellence, 26 were treated with natalizumab for breakthrough disease. ${ }^{93}$ The medication appears to be well tolerated and further, appears to have resulted in improved disease control. Of children started on natalizumab, only three changed therapies subsequently: one experienced a hypersensitivity reaction, one discontinued due to breakthrough disease one month after initiation, and one discontinued the medication due to side effects (gastrointestinal upset). There have been no reports to date of PML in association with this young population.

\section{Mitoxantrone}

Mitoxantrone (MITO) is an anthracendione cytotoxic agent with immunosuppressive properties. Safety and efficacy studies have been performed. ${ }^{94,95}$ Based on the results of a phase III study of 194 patients randomized to two treatment groups (MITO $12 \mathrm{vs} 5 \mathrm{mg} / \mathrm{m}^{2}$ ) and one placebo group, MITO was approved in the US and Europe for 
the treatment of worsening RRMS and SPMS in adults. ${ }^{96}$ MITO is structurally related to anthracycline agents with well-established cardiotoxicity. Long-term clinical use of MITO requires caution; the lifetime cumulative dosage should not exceed $140 \mathrm{mg} / \mathrm{m}^{2}$ Left ventricular ejection fraction evaluation (MUGA or ECHO) should be performed before each MITO infusion and monitoring of blood counts should be performed regularly because of an increased risk for treatment-related acute leukemia. Importantly, recent data point to a higher than previously reported dose-dependent risk (greater in patients receiving $>60 \mathrm{mg} / \mathrm{m}^{2}$ ) for acute leukemia in patients who have received mitoxantrone. ${ }^{97,98}$

There have been no published reports of mitoxantrone use in the pediatric population. Twelve of 258 patients followed at the US Pediatric MS Centers of Excellence received mitoxantrone..$^{77}$ No major side effects have been noted. However, we suggest caution with its use, given the potential for major side effects with this drug.

\section{Rituximab}

B cells, immunoglobulins and complement are increasingly being implicated in the pathogenesis of MS. ${ }^{15}$ Rituximab is a B cell-depleting chimeric monoclonal antibody against the protein CD20. It was originally used in chemotherapeutic regimens for the treatment of non-Hodgkin's lymphoma. It has been used with success in multiple autoimmune diseases, such as rheumatoid arthritis, idiopathic thrombocytopenic purpura (ITP), systemic lupus erythematosus, and Sjögren's among others.

A phase-II, double-blind, placebo-controlled trial evaluating response in adult-onset RRMS to two infusions of rituximab, given on days 1 and 15, suggested a decrease in inflammatory brain lesions and clinical relapses over a 48 -week period. At 24 weeks, $14.5 \%$ of patients in the rituximab group had relapses compared with $40 \%$ in the placebo group. At week 48, the number of patients was $20 \%$ vs $40 \%$. Side effects were common; $98 \%$ of patients on rituximab vs $35 \%$ on placebo reported side effects. The number of patients experiencing serious adverse events was similar in the placebo (14.3\%) and rituximab (13.0\%) groups. It is therefore a promising therapy for patients with active RRMS. ${ }^{45,46}$

PML has been reported in association with rituximab. The FDA recently issued a warning after two patients with SLE developed PML while on therapy with rituximab. However, no cases to date have been reported in association with its use in MS.
In the pediatric MS population, only one case report of rituximab has been published; a dramatic decrease in relapse number was reported in an adolescent with severe RRMS. The effect lasted for 2 years from the time of initiation of therapy. ${ }^{99}$

\section{Cyclophosphamide}

Cyclophosphamide (CTX), an alkylating agent with potent cytotoxic and immunosuppressive effects, has been studied in controlled, unblinded trials using high-doses IV as an induction therapy or in repeated cycles; these regimens have shown benefit in patients with SPMS in some, but not all studies. ${ }^{94,95} \mathrm{CTX}$ has multiple side effects including leukopenia, myocarditis, hemorrhagic cystitis, and alopecia. Therefore, some consider CTX to be a treatment for adult RRMS patients with active disease, who have failed less toxic therapies or have a rapidly progressive course.

One case series describing a cohort of children with highly inflammatory and aggressive RRMS, in whom CTX was initiated, has been published. ${ }^{100}$ All children experienced multiple relapses in the year prior to initiation of CTX despite being treated with a first-line therapy (average annualized relapse rate of 3.8). Further, physical disability in this cohort was marked. Almost one-fourth of the cohort had an EDSS of 6.0 or higher. Half of the children who received CTX in this study later required combination therapy or treatment with another second-line agent. Only one-third of the children were able to go back to a first-line therapy. With regards to tolerability, almost all children receiving CTX experienced side effects, some of which were serious, including ITP, infertility, osteoporosis, and transitional cell carcinoma. This therapy should therefore be used with caution in this population.

\section{Future therapies: oral agents}

Phase III clinical trials have been published on two oral agents in adult MS, fingolimod, and cladribine..$^{52,53,101}$ Both of these agents target lymphocytes through different mechanisms of action.

Fingolimod is a sphingosine 1-phosphate receptor modulator. It prevents the egress of lympohcytes from lymph nodes. Two pivotal phase III, randomized controlled trials, one a placebo-controlled trial and the other a comparison of fingolimod to interferon $\beta$ - $1 \mathrm{a}$, showed efficacy of oral fingolimod over placebo at doses of 0.5 or $1.25 \mathrm{mg}$ daily. The placebo-controlled trial showed a significant reduction in annualized relapse rate $(0.18$ [0.5 mg], 0.16 [1.25 mg], 
0.4 [placebo], $P<0.001$ ), risk of disability progression, and MRI measures of atrophy and lesion count in patients on fingolimod. ${ }^{53}$

In the trial comparing fingolimod with interferon $\beta$-1a IM, patients on fingolimod had a significantly lower annualized relapse rate than those on interferon $(0.2[1.25 \mathrm{mg}]$, 0.16 [0.5 mg] vs 0.33 [interferon], $P<0.001)$. Adverse events in both trials included elevated LFTs, macular edema, skin cancer, herpes virus infections (including two fatal infections at the $1.25 \mathrm{mg}$ dose), hypertension, and cardiac arrhythmias (bradycardia, atrioventricular conduction block). ${ }^{51,53}$

Cladribine is an immunomodulatory agent that targets lympohcyte subsets. Two dosing regimens were evaluated in a pivotal phase III trial: 3.5 and $5.25 \mathrm{mg}$ per kilogram body weight. In this large, randomized, placebo-controlled, double-blind trial, in comparison to patients on placebo, patients on cladribine were found to have a significant reduction in annualized relapse rate $(0.14$ [3.5 mg/kg], 0.15 [5.25 mg/kg], 0.33 [placebo], $P<0.001)$ and MRI lesion count. Patients on cladribine were also found to have higher relapse-free rate than those on placebo $(79.7 \%[3.5 \mathrm{mg} / \mathrm{kg}]$, $78.9 \%$ [5.25 mg], 60.9\% [placebo], $P<0.001)$. The most significant complications were those of lymphocytopenia and herpes zoster. ${ }^{52}$

No studies of the use of these agents in the pediatric population have been published. However, given the serious adverse events reported in association with these agents in the adult population, including cancer and lethal herpetic infections, caution should be taken before adopting these therapies for the pediatric population.

\section{Conclusion}

A growing body of literature has helped to provide better guidelines for the diagnosis and treatment of pediatric MS. Pediatric and adolescent MS have been shown to carry a greater disease burden than adult MS, both in terms of annualized relapse rate and MRI parameters. First-line therapies currently used in the adult population appear to be safe in children with MS. Breakthrough disease requiring a change in therapy is relatively common in this population. Current second-line therapies used in the adult population have been used in a small number of children with MS and appear to be tolerated. Oral agents have not been evaluated in children with MS. Future studies concentrating on the early diagnosis of MS are needed as are those evaluating the role of therapies approved for use in the adult MS population in children with MS.

\section{Disclosure}

Dr Yeh has received research support from the National Institutes of Health (Co-I: R01 NS062820-01 A2), the Jog for the Jake Foundation, and the Children's Guild Foundation. Her clinical activities are supported in part by a National MS Society Pediatric MS Center of Excellence grant. She serves as a consultant on the Peripheral and Central Nervous System Drugs Advisory Committee (PCNS) of the FDA.

\section{References}

1. Boiko A, Vorobeychik G, Paty D, Devonshire V, Sadovnick D. Early onset multiple sclerosis: a longitudinal study. Neurology. 2002;59(7): 1006-1010.

2. Chitnis T, Glanz B, Jaffin S, Healy B. Demographics of pediatric-onset multiple sclerosis in an MS center population from the Northeastern United States. Mult Scler (Houndmills, Basingstoke, England). 2009; 15(5):627-631.

3. Ghezzi A, Deplano V, Faroni J, et al. Multiple sclerosis in childhood: clinical features of 149 cases. Mult Scler (Houndmills, Basingstoke, England). 1997;3(1):43-46.

4. Renoux C, Vukusic S, MikaeloffY, et al. Natural history of multiple sclerosis with childhood onset. N Engl J Med. 2007;356(25):2603-2613.

5. Gorman MP, Healy BC, Polgar-Turcsanyi M, Chitnis T. Increased relapse rate in pediatric-onset compared with adult-onset multiple sclerosis. Arch Neurol. 2009;66(1):54-59.

6. Waubant E, Chabas D, Okuda DT, et al. Difference in disease burden and activity in pediatric patients on brain magnetic resonance imaging at time of multiple sclerosis onset vs adults. Arch Neurol. 2009;66(8): 967-971.

7. Yeh EA, Weinstock-Guttman B, Ramanathan M, et al. Magnetic resonance imaging characteristics of children and adults with paediatric-onset multiple sclerosis. Brain. 2009;132(Pt 12):3392-3400.

8. Mikaeloff Y, Adamsbaum C, Husson B, et al. MRI prognostic factors for relapse after acute CNS inflammatory demyelination in childhood. Brain. 2004;127(Pt 9):1942-1947.

9. Mikaeloff Y, Caridade G, Husson B, Suissa S, Tardieu M. Acute disseminated encephalomyelitis cohort study: prognostic factors for relapse. Eur J Paediatr Neurol. 2007;11(2):90-95.

10. Krupp LB, Banwell B, Tenembaum S. Consensus definitions proposed for pediatric multiple sclerosis and related disorders. Neurology. 2007;68(16 Suppl 2):S7-S12.

11. Hahn CD, Shroff MM, Blaser SI, Banwell BL. MRI criteria for multiple sclerosis: evaluation in a pediatric cohort. Neurology. 2004; 62(5):806-808.

12. Callen DJ, Shroff MM, Branson HM, et al. Role of MRI in the differentiation of ADEM from MS in children. Neurology. 2009; 72(11):968-973.

13. Callen DJ, Shroff MM, Branson HM, et al. MRI in the diagnosis of pediatric multiple sclerosis. Neurology. 2009;72(11):961-967.

14. Ketelslegers IA, Neuteboom RF, Boon M, Catsman-Berrevoets CE, Hintzen RQ. A comparison of MRI criteria for diagnosing pediatric ADEM and MS. Neurology. 2010;74(18):1412-1415.

15. Lucchinetti C, Bruck W, Parisi J, Scheithauer B, Rodriguez M, Lassmann H. Heterogeneity of multiple sclerosis lesions: implications for the pathogenesis of demyelination. Ann Neurol. 2000;47(6): 707-717.

16. Callen DJ, Shroff MM, Branson HM, et al. Role of MRI in the differentiation of ADEM from MS in children. Neurology. 2008.

17. Young NP, Weinshenker BG, Parisi JE, et al. Perivenous demyelination: association with clinically defined acute disseminated encephalomyelitis and comparison with pathologically confirmed multiple sclerosis. Brain. 133(Pt 2):333-348.

18. Bar-Or A. The immunology of multiple sclerosis. Semin Neurol. 2008; 28(1):29-45. 
19. Berger T, Rubner P, Schautzer F, et al. Antimyelin antibodies as a predictor of clinically definite multiple sclerosis after a first demyelinating event. N Engl J Med. 2003;349(2):139-145.

20. O’Connor KC, Lopez-Amaya C, Gagne D, et al. Anti-myelin antibodies modulate clinical expression of childhood multiple sclerosis J Neuroimmunol. 2010;223(1-2):92-99.

21. Chabas D, Ness J, Belman A, et al. Younger children with MS have a distinct CSF inflammatory profile at disease onset. Neurology. 74(5): 399-405.

22. Brusaferri F, Candelise L. Steroids for multiple sclerosis and optic neuritis: a meta-analysis of randomized controlled clinical trials. J Neurol. 2000;247(6):435-442.

23. Beck RW. The optic neuritis treatment trial: three-year follow-up results. Arch Ophthalmol. 1995;113(2):136-137.

24. Alam SM, Kyriakides T, Lawden M, Newman PK. Methylprednisolone in multiple sclerosis: a comparison of oral with intravenous therapy at equivalent high dose. J Neurol Neurosurg Psychiatry. 1993; 56(11):1219-1220.

25. Burton JM, O’Connor PW, Hohol M, Beyene J. Oral versus intravenous steroids for treatment of relapses in multiple sclerosis. Cochrane Database Syst Rev. 2009;(3):CD006921.

26. Waldman A, Gorman M, Rensel M, et al. Pediatric CNS demyelinating disorders: survey of US practice patterns. Manuscript.

27. Fazekas F, Deisenhammer F, Strasser-Fuchs S, Nahler G, Mamoli B. Treatment effects of monthly intravenous immunoglobulin on patients with relapsing-remitting multiple sclerosis: further analyses of the Austrian Immunoglobulin in MS study. Mult Scler (Houndmills, Basingstoke, England). 1997;3(2):137-141.

28. Sorensen PS, Wanscher B, Schreiber K, Blinkenberg M, Jensen CV, Ravnborg M. A double-blind, cross-over trial of intravenous immunoglobulin G in multiple sclerosis: preliminary results. Mult Scler (Houndmills, Basingstoke, England). 1997;3(2):145-148.

29. Achiron A, Gabbay U, Gilad R, et al. Intravenous immunoglobulin treatment in multiple sclerosis. Effect on relapses. Neurology. 1998; 50(2):398-402.

30. Sorensen PS, Fazekas F, Lee M. Intravenous immunoglobulin G for the treatment of relapsing-remitting multiple sclerosis: a meta-analysis. Eur J Neurol. 2002;9(6):557-563.

31. Fazekas F, Lublin FD, Li D, et al. Intravenous immunoglobulin in relapsing-remitting multiple sclerosis: a dose-finding trial. Neurology. 2008;71(4):265-271.

32. Pohlau D, Przuntek H, Sailer M, et al. Intravenous immunoglobulin in primary and secondary chronic progressive multiple sclerosis: a randomized placebo controlled multicentre study. Mult Scler (Houndmills, Basingstoke, England). 2007;13(9):1107-1117.

33. Visser LH, Beekman R, Tijssen CC, et al. A randomized, double-blind, placebo-controlled pilot study of i.v. immune globulins in combination with i.v. methylprednisolone in the treatment of relapses in patients with MS. Mult Scler (Houndmills, Basingstoke, England). 2004; 10(1):89-91.

34. Sorensen PS, Haas J, Sellebjerg F, Olsson T, Ravnborg M. IV immunoglobulins as add-on treatment to methylprednisolone for acute relapses in MS. Neurology. 2004;63(11):2028-2033.

35. Tselis A, Perumal J, Caon C, et al. Treatment of corticosteroid refractory optic neuritis in multiple sclerosis patients with intravenous immunoglobulin. Eur J Neurol. 2008;15(11):1163-1167.

36. Straussberg R, Schonfeld T, Weitz R, Karmazyn B, Harel L. Improvement of atypical acute disseminated encephalomyelitis with steroids and intravenous immunoglobulins. Pediatr Neurol. 2001; 24(2):139-143.

37. Spalice A, Properzi E, Lo Faro V, Acampora B, Iannetti P. Intravenous immunoglobulin and interferon: successful treatment of optic neuritis in pediatric multiple sclerosis. J Child Neurol. 2004;19(8):623-626.

38. Shahar E, Andraus J, Savitzki D, Pilar G, Zelnik N. Outcome of severe encephalomyelitis in children: effect of high-dose methylprednisolone and immunoglobulins. J Child Neurol. 2002;17(11):810-814.

39. Rodriguez M, Karnes WE, Bartleson JD, Pineda AA. Plasmapheresis in acute episodes of fulminant CNS inflammatory demyelination. Neurology. 1993;43(6):1100-1104.
40. Llufriu S, Castillo J, Blanco Y, et al. Plasma exchange for acute attacks of CNS demyelination: predictors of improvement at 6 months. Neurology. 2009;73(12):949-953.

41. Schilling S, Linker RA, Konig FB, et al. Plasma exchange therapy for steroid-unresponsive multiple sclerosis relapses: clinical experience with 16 patients. Nervenarzt. 2006;77(4):430-438.

42. Takahashi I, Sawaishi Y, Takeda O, Enoki M, Takada G. Childhood multiple sclerosis treated with plasmapheresis. Pediatr Neurol. 1997; 17(1):83-87.

43. Amato MP, Goretti B, Ghezzi A, et al. Cognitive and psychosocial features of childhood and juvenile MS. Neurology. 2008;70(20): 1891-1897.

44. MikaeloffY, Caridade G, Tardieu M, Suissa S. Effectiveness of early $\beta$ interferon on the first attack after confirmed multiple sclerosis: a comparative cohort study. Eur J Paediatr Neurol. 2008;12(3): 205-209.

45. Hauser SL, Waubant E, Arnold DL, et al. B-cell depletion with rituximab in relapsing-remitting multiple sclerosis. $N$ Engl $\mathrm{J} \mathrm{Med}$. 2008;358(7):676-688

46. Bar-Or A, Calabresi PA, Arnold D, et al. Rituximab in relapsingremitting multiple sclerosis: a 72-week, open-label, phase I trial. Ann Neurol. 2008;63(3):395-400.

47. Bielekova B, Richert N, Howard T, et al. Humanized anti-CD25 (daclizumab) inhibits disease activity in multiple sclerosis patients failing to respond to interferon $\beta$. Proc Natl Acad Sci U S A. 2004;101(23):8705-8708.

48. Krishnan C, Kaplin AI, Brodsky RA, et al. Reduction of disease activity and disability with high-dose cyclophosphamide in patients with aggressive multiple sclerosis. Arch Neurol. 2008;65(8): 1044-1051.

49. Then Bergh F, Kumpfel T, Schumann E, et al. Monthly intravenous methylprednisolone in relapsing-remitting multiple sclerosis - reduction of enhancing lesions, T2 lesion volume and plasma prolactin concentrations. BMC Neurol. 2006;6:19.

50. Fazekas F, Deisenhammer F, Strasser-Fuchs S, Nahler G, Mamoli B. Randomised placebo-controlled trial of monthly intravenous immunoglobulin therapy in relapsing-remitting multiple sclerosis. Austrian Immunoglobulin in Multiple Sclerosis Study Group. Lancet. 1997; 349(9052):589-593.

51. Cohen JA, Barkhof F, Comi G, et al. Oral fingolimod or intramuscular interferon for relapsing multiple sclerosis. $N$ Engl J Med. 362(5): $402-415$.

52. Giovannoni G, Comi G, Cook S, et al. A placebo-controlled trial of oral cladribine for relapsing multiple sclerosis. $N$ Engl J Med. 362(5): 416-426.

53. Kappos L, Radue EW, O'Connor P, et al. A placebo-controlled trial of oral fingolimod in relapsing multiple sclerosis. $N$ Engl J Med. 362(5): $387-401$.

54. The IFNB Multiple Sclerosis Study Group. Interferon $\beta$ - $1 \mathrm{~b}$ is effective in relapsing-remitting multiple sclerosis. I Clinical results of a multicenter, randomized, double blind, placebo-controlled trial. Neurology. 1993:655-661.

55. Jacobs L, Cookfair D, Rudick R, et al. Intramuscular Interferon $\beta$-1a for disease progression in relapsing multiple sclerosis. Ann Neurol. 1996;39:285-294.

56. PRISMS (Prevention of Relapses and Disability by Interferon $\beta$-1a Subcutaneously in Multiple Sclerosis) Study Group. Randomized double-blind placebo-controlled study of interferon $\beta$-1a in relapsing/remitting multiple sclerosis. Lancet. 1998;352: 1498-1504.

57. Tenembaum SN, Segura MJ. Interferon $\beta$-1a treatment in childhood and juvenile-onset multiple sclerosis. Neurology. 2006;67(3): 511-513.

58. Mikaeloff $\mathrm{Y}$, Moreau $\mathrm{T}$, Debouverie $\mathrm{M}$, et al. Interferon- $\beta$ treatment in patients with childhood-onset multiple sclerosis. $J$ Pediatr. 2001;139(3):443-446.

59. Banwell B, Reder AT, Krupp L, et al. Safety and tolerability of interferon $\beta-1 \mathrm{~b}$ in pediatric multiple sclerosis. Neurology. 2006; 66(4):472-476. 
60. Bykova OV, Kuzenkova LM, Maslova OI. The use of $\beta$-interferon- $1 \mathrm{~b}$ in children and adolescents with multiple sclerosis. Zh Nevrol Psikhiatr Im S S Korsakova. 2006;106(9):29-33.

61. Pohl D, Rostasy K, Gartner J, Hanefeld F. Treatment of early onset multiple sclerosis with subcutaneous interferon $\beta$-1a. Neurology. 2005;64(5):888-890.

62. Waubant E, Hietpas J, Stewart T, et al. Interferon $\beta$-1a in children with multiple sclerosis is well tolerated. Neuropediatrics. 2001; 32(4):211-213.

63. Yeh E, Waubant E, Krupp L, et al. Breakthrough disease in pediatric MS patients. Manuscript. 2010.

64. Ghezzi A, Amato MP, Capobianco M, et al. Treatment of early-onset multiple sclerosis with intramuscular interferon $\beta$-1a: long-term results. Neurol Sci. 2007;28(3):127-132.

65. Pittock SJ. Does benign multiple sclerosis today imply benign multiple sclerosis tomorrow? Implications for treatment. Neurology. 2007; 68(7):480-481.

66. Ramsaransing GS, De Keyser J. Benign course in multiple sclerosis: a review. Acta Neurol Scand. 2006;113(6):359-369.

67. Pittock SJ, McClelland RL, Mayr WT, et al. Clinical implications of benign multiple sclerosis: a 20 -year population-based follow-up study. Ann Neurol. 2004;56(2):303-306.

68. Malucchi S, Sala A, Gilli F, et al. Neutralizing antibodies reduce the efficacy of $\beta$ IFN during treatment of multiple sclerosis. Neurology. 2004;62(11):2031-2037.

69. Perini P, Calabrese M, Biasi G, Gallo P. The clinical impact of interferon $\beta$ antibodies in relapsing-remitting MS. J Neurol. 2004; 251(3):305-309.

70. Dhib-Jalbut S. Sustained immunological effects of Glatiramer acetate in patients with multiple sclerosis treated for over 6 years. $J$ Neurol Sci. 2002;201:71-77.

71. Johnson KP, Brooks BR, Cohen JA, et al. Copolymer 1 reduces relapse rate and improves disability in relapsing-remitting multiple sclerosis: results of a phase III multicenter, double-blind placebo-controlled trial. The Copolymer 1 Multiple Sclerosis Study Group. Neurology. 1995; 45(7): 1268-1276.

72. Comi G, Filippi M, Wolinsky JS. European/Canadian multicenter, double-blind, randomized, placebo-controlled study of the effects of glatiramer acetate on magnetic resonance imaging - measured disease activity and burden in patients with relapsing multiple sclerosis. European/ Canadian Glatiramer Acetate Study Group. Ann Neurol. 2001;49(3): 290-297.

73. Cadavid D, Wolansky LJ, Skurnick J, et al. Efficacy of treatment of MS with IFN $\beta-1 b$ or glatiramer acetate by monthly brain MRI in the BECOME study. Neurology. 2009;72(23):1976-1983.

74. Ghezzi A. Immunomodulatory treatment of early onset multiple sclerosis: results of an Italian Co-operative Study. Neurol Sci. 2005;26 Suppl 4:S183-S186.

75. Ghezzi A, Amato MP, Capobianco M, et al. Disease-modifying drugs in childhood-juvenile multiple sclerosis: results of an Italian co-operative study. Mult Scler (Houndmills, Basingstoke, England). 2005;11(4):420-424.

76. Kornek B, Bernert G, Balassy C, Geldner J, Prayer D, Feucht M. Glatiramer acetate treatment in patients with childhood and juvenile onset multiple sclerosis. Neuropediatrics. 2003;34(3): 120-126.

77. Yeh E, Krupp L, Ness J, et al. Breakthrough disease in pediatric MS patients: a pediatric network experience. Annual Meeting of the American Academy of Neurology. Seattle, WA: 2009.

78. Cohen BA, Khan O, Jeffery DR, et al. Identifying and treating patients with suboptimal responses. Neurology. 2004;63(12 Suppl 6):S33-S40.

79. Panitch H, Goodin D, Francis G, et al. Randomized, comparative study of interferon $\beta$-1a treatment regimens in MS; The EVIDENCE Trial. Neurology. 2002;359:1453-1460.
80. Durelli L, Verdun E, Barbero P, et al. Every other day interferon $\beta-1 b$ versus once-weekly interferon $\beta$-1a for multiple sclerosis; results of a 2 -year prospective randomized multicenter study (INCOMIN). Lancet. 2002;359:1453-1460.

81. Group. IMSSGatUoBCMMA. Neutralizing antibodies during treatment of multiple sclerosis with interferon $\beta-1 \mathrm{~b}$ : experience during the first 3 years. Neurology. 1996;47:889-894.

82. Sorensen P, Ross C, Clemmesen K, et al. Clinical importance of neutralizing antibodies against interferon $\beta$ in patients with relapsing remitting multiple sclerosis. Lancet. 2003;302:1184-1191.

83. Archelos J, Pervitali S, Hartung H. The role of integrins in immune-mediated diseases of the nervous system. Trends Neurosci. 1999;22:30-38.

84. Miller D, Khan O, Sheremata W, et al. A controlled trial of natalizumab for relapsing multiple sclerosis. N Engl J Med. 2003;348:15-23.

85. Volmer T, Phillips J, goodman A, et al. An open label safety and drug interaction study of natalizumab (Antegran) in combination with IFN $\beta$ in patients with MS. Mult Scler (Houndmills, Basingstoke, England). 2004;10(5):511-520.

86. Rudick RA, Stuart WH, Calabresi PA, et al. Natalizumab plus interferon $\beta$-1a for relapsing multiple sclerosis. $N$ Engl J Med. 2006;354(9):911-923.

87. Polman $\mathrm{CH}, \mathrm{O}$ 'Connor PW, Havrdova E, et al. A randomized, placebo-controlled trial of natalizumab for relapsing multiple sclerosis. N Engl J Med. 2006;354(9):899-910.

88. VanAssche G, Ranst MV, Sciot R, et al. Progressive multifocal leukoencephalopathy after natalizumab therapy for Crohn's disease. N Engl J Med. 2005;353:362-368.

89. Langer-Gould A, Atlas S, Green A, et al. Progressive multifocal encephalopathy in a patient treated with natalizumab. $N$ Engl J Med. 2005;353:375-381.

90. Kleinschmidt-DeMasters B, Tyler K. Progressive multifocal leukoencphalopathy complicating treatment with natalizumab and interferon $\beta$-1a for multiple sclerosis. $N$ Engl J Med. 2005;353: 369-374.

91. Borriello G, Prosperini L, Luchetti A, Pozzilli C. Natalizumab treatment in pediatric multiple sclerosis: a case report. Eur J Paediatr Neurol. 2009;13(1):67-71.

92. Huppke P, Stark W, Zurcher C, Huppke B, Bruck W, Gartner J. Natalizumab use in pediatric multiple sclerosis. Arch Neurol. 2008;65(12):1655-1658

93. Yeh E, Kuntz N, Chabas D, et al. Use of natalizumab in pediatric MS: a collaborative network study. Child Neurology Society (Submitted). Louisville, KY: 2009.

94. Weiner $\mathrm{H}$, ackin $\mathrm{G}$, Orav E, et al. Intermittent cyclophosphamide pulse therapy in progressive multiple sclerosis: final report of the Northeast Cooperative Multiple Sclerosis Treatment Group. Neurology. 1993; 43:910-918.

95. The Canadian Cooperative Multiple Sclerosis Study Group. The Canadian cooperative trial of cyclophosphamide and plasma exchange in progressive multiple sclerosis. Lancet. 1991; 337:441-446.

96. Hartung H, Gonsette R, Konig N, et al; Group tM-S. Mitoxantrone in progressive multiple sclerosis: a placebo-controlled, double-blind, randomized, multicenter trial. Lancet. 2002;360:2018-2025.

97. Ellis R, Boggild M. Therapy-related acute leukaemia with Mitoxantrone: what is the risk and can we minimise it? Mult Scler (Houndmills, Basingstoke, England). 2009;15(4):505-508.

98. Pascual AM, Tellez N, Bosca I, et al. Revision of the risk of secondary leukaemia after mitoxantrone in multiple sclerosis populations is required. Mult Scler (Houndmills, Basingstoke, England). 2009;15(11): 1303-1310.

99. Karenfort M, Kieseier BC, Tibussek D, Assmann B, Schaper J, Mayatepek E. Rituximab as a highly effective treatment in a female adolescent with severe multiple sclerosis. Dev Med Child Neurol. 2009;51(2):159-161. 
100. Makhani N, Gorman MP, Branson HM, Stazzone L, Banwell BL, Chitnis T. Cyclophosphamide therapy in pediatric multiple sclerosis. Neurology. 2009;72(24):2076-2082.
101. Chun J, Hartung HP. Mechanism of action of oral fingolimod (FTY720) in multiple sclerosis. Clin Neuropharmacol. 2010;33(2):91-101.

\section{Publish your work in this journal}

Adolescent Health, Medicine and Therapeutics is an international, peer-reviewed, open access journal focusing on health, pathology, and treatment issues specific to the adolescent age group. All aspects of health maintenance, preventative measures and disease treatment interventions are addressed within the journal and practitioners from all disciplines are invited to submit their work as well as healthcare researchers and patient support groups.. The manuscript management system is completely online and includes a very quick and fair peerreview system. Visit http://www.dovepress.com/testimonials.php to read real quotes from published authors.

Submit your manuscript here: http://www.dovepress.com/adolescent-health-medicine-and-therapeutics-journal 